\title{
VERDAD LÓGICA, ANALITICIDAD Y CONVENCIONALISMO EN CARNAP
}

\section{Introducción}

El concepto carnapiano de verdad lógica ("Verdad-L", en su terminologla esotérica) se presenta como una elucidación de "lo que Leibniz llamaba verdad necesaria y Kant verdad analítica" (Meaning and Necessity, p. 11; en adelante, " $M \& \&$ \&".). Puede ser útil, pues, una breve digresión histórica.

La caracterización leibniziana de verdad necesaria coincide en general con la definición kantiana de analiticidad, pues según Leibniz toda verdad necesaria es reducible a una identidad parcial o total. Pero Kant y Leibniz no atribuyen la misma extensión a estos conceptos. Hablando en términos kantianos, Leibniz diría que lo analítico agota el dominio de las verdades necesarias. Ésta es la tesis del positivismo lógico, que niega la existencia de la síntesis a priori. En su forma logicista pura, esta tesis afirma que todo lo a priori es analítico e identifica el dominio de lo analítico con el de la lógica, cuyas leyes son concebidas como puramente 'formales'. En su versión positivista, el carácter 'formal' no sólo despoja a las leyes lógicas de todo contenido empírico sino de cualquier implicación ontológica, por general que fuere; son necesarias, si, pero al precio de no proveer información alguna acerca del mundo, ni siquiera de carácter estructural. En su tiempo, Kant habia impugnado las pretensiones de la metafísica dogmática con un diagnóstico audaz: "Sólo podemos conocer a priori en las cosas lo que nosotros mismos hemos puesto en ellas" (Introd. a la $2^{\mathrm{a}}$ ed. de la Crítica de la razón pura); lo que la razón descubre por sí sola no nos revela la naturaleza del universo sino la anatomía del sujeto cognoscente. El positivismo lógico no habla de formas puras de la sensibilidad y categorias del entendimiento, sino de convenciones lingüísticas o reglas semánticas: las verdades a priori no nos muestran la estructura del mundo (y menos aún hechos particulares sobre él) sino la anatomfa de nuestro lenguaje; no son un alarde del poder ilimitado de la razón, sino los límites que las reglas lingüísticas imponen a nuestro pensamiento.

En la historia de las relaciones entre lo a priori, lo analitico y lo sintético, importa distinguir con claridad dos momentos: aquellos en los que se produce un cambio de tesis acerca de tales relaciones, y aquellos en los que se asiste a un cambio de sentido de estos términos claves. Aunque ambas cosas pueden darse en forma combinada, sólo su distinción evitará que el proceso se muestre envuelto en una niebla imbatible. 
Kant dividió el campo de las verdades necesarias entre los juicios analíticos y los sintéticos a priori; después de él, Frege preserva la misma división, pero sostiene, contrariamente a Kant, que la aritmética es analítica (si bien la geometría sigue siendo sintética). Asistimos, al parecer, a un enfrentamiento de tesis opuestas; pero la claridad del diálogo se enturbia apenas advertimos que se produjo también un cambio brusco en la noción misma de analiticidad. Frege define como analítico lo que es o bien una ley lógica o puede derivarse a partir de leyes lógicas mediante definiciones y reglas de inferencia; pero Frege no entendía por "lógica" lo mismo que Kant, para quien, sin embargo, también la lógica era analítica. La de Frege es una lógica ampliada de modo revolucionario, en cuyos dominios la forma Sujeto-Predicado, de la que depende la definición kantiana de analiticidad, ocupa un lugar modesto; incluye además los axiomas conjuntísticos necesarios para la derivación de la aritmética, axiomas que Kant no hubiera considerado nunca como parte de la lógica.

Kant afirma, pues, que la aritmética es sintética a priori; Frege responde que en realidad es analítica. ¿Hasta qué punto se contradicen? No vamos a examinar esta cuestión aquí; baste señalar que no hablan exactamente el mismo lenguaje: ni "analftico" ni "sintético" significan lo mismo para ambos.

Un instante curioso de esta historia puede encontrarse en el libro de Bertrand Russell The problems of philosophy (1912), donde Russell acredita a Kant el mérito de "haberse dado cuenta" de que las proposiciones de la aritmética y la geometria son sintéticas a priori. The problems of philosophy se publicó dos años después del primer volumen de Principia Mathematica, obra celebrada por los positivistas como el triunfo final y la gloria del logicismo, pues probaba - según se suponía - la reducibilidad de la aritmética a la lógica, y lo hacía sin caer en las paradojas que afectaban el sistema de Frege y pusieron en duda la posibilidad misma de la empresa. ¿Cómo se explica, entonces, que Russell le dé la razón a Kant? La respuesta carece (ןay!) de todo misterio: Russell se mantenfa fiel, aun después de Principia, a la definición kantiana de analítico; nos dice simplemente que en las proposiciones matemáticas "el análisis del sujeto no puede revelar el predicado" y que Kant probó esto "con perfecta exactitud", tomando como ejemplo la ecuación " $7+5=12$ ".

Cuando Wittgenstein presentó en el Tractatus la noción de 'tautologfa' Russell volvió sobre sus pasos: pensó que el carácter tautológico representaba mejor cierta propiedad distintiva de las proposiciones lógicas que solía indicarse llamándolas analíticas, y deseó — para resolver dificultades propias de su sistema- que las verdades matemáticas fueran también tautologłas. ${ }^{1}$ La definición kantiana había sido una caracterización deficiente de una intui-

1 Cfr. Introduction to Mathematical Philosophy (1919), p. 202, y la introducción a la $2^{2}$ ed. de Principia Mathematica (1927), p. xiv. 
ción certera. Con palabras de Wittgenstein: "Las proposiciones de la lógica son tautologias. Por lo tanto no dicen nada (son las proposiciones analíticas)" (Tractatus, 6.1-6.11). Junto con la tesis de que las verdades de la lógica y de la matemática son tautologías se introdujo, pues, la identificación de lo analítico con lo tautológico, y por lo tanto una nueva noción de analiticidad.

En la obra de Carnap este doble proceso se manifiesta con matices desconcertantes, algunos de los cuales serán discutidos aqui. No es nuestro propósito seguir sus huellas desde los pasos iniciales; nuestro examen girará esencialmente en torno de las tesis expuestas por Carnap en su libro Meaning and Necessity, que fue identificado alguna vez con "la edad de oro de la semántica". Para una comprensión más clara de lo que sigue es conveniente distinguir en Carnap tres aspectos distintos:

i) La tesis de que existe una distinción neta entre enunciados analíticos y sintéticos, distinción que constituye una herramienta conceptual indispensable;

ii) La elucidación carnapiana de esta distinción;

iii) Las posturas convencionalistas asociadas con (i) y (ii).

Parece claro que (i), (ii) y (iii) son independientes; nosotros nos ocuparemos de (ii) y (iii), pero omitiremos toda discusión básica sobre (i).

Respecto de (iii) es pertinente también una breve aclaración. El método de análisis de Carnap consiste, grosso modo, en la construcción de modelos semánticos cuyas reglas explícitas sugieren con fuerza una actitud convencionalista, pues - con ciertas reservas- su autor es el dios de sus sistemas y puede decidir acerca de ellas; pero el método de modelización —aplicado por otros autores en el estudio de la gramática de los lenguajes naturales- no guarda con el convencionalismo una relación necesaria.

\section{Carnap: definición y criterio de adecuación. Efectividad de las reglas seminticas}

Antes de formular su definición de verdad lógica, Carnap presenta un criterio de adecuación que debe ser satisfècho por toda definición de ese concepto; pues su propósito no es ofrecer una definición estipulativa, sino una elucidación [explication] de un concepto existente en la tradición filosófica: el de "verdad lógica o necesaria o analítica" (loc. cit., p. 10).

Según nos explica, el concepto de verdad necesaria (para escoger uno de los términos) "fue caracterizado a veces como verdad basada en razones puramente logicas, en el significado solamente", con independencia de los hechos empíricos (p. 10). Y es esta idea intuitiva la que debe captar la definición que propone. Aplicando su peculiar método elucidatorio, formula su definición para un lenguaje artificial o sistema semántico especifico, que puede concebirse como un modelo simplificado del lenguaje natural; y en un sistema 
semántico "el significado de una oración, su interpretación, está determinada [explícitamente (T. M. S.)] por-las reglas semánticas" (p. 10)..$^{2}$ Es por eso que el criterio de adecuación toma la forma de la

CONVEnctón 2.r: Una oración $A$ es L-verdadera en un sistema semántico $S$ si y sólo si es verdadera en $S$ de modo tal que su verdad puede establecerse sobre la base de las reglas semánticas de $S$ solamente, sin ninguna referencia a hechos extralingüisticos. ${ }^{3}$

Dicho en forma algo más simple: una oración es lógicamente verdadera si y sólo si: a) es verdadera, y b) las reglas semánticas bastan para establecerlo. Queda por hallar una definición que satisfaga este requerimiento.

Antes de presentarla debemos introducir dos nociones: la de 'descripción de estado' y la de 'valer en una descripción de estado'. Una descripción de estado $D$ es una clase de oraciones que posee la siguiente propiedad: dada cualquier oración atómica, pertenecen a $D$ o bien ella o su negación, pero no ambas, y $D$ no contiene ningún otro tipo de oraciones. No daremos aquí la definición exacta de 'valer en una descripción de estado'; baste decir, en términos informales, que una oración vale en una descripción de estado $D$ si sería verdadera en el caso de que lo fuese $D$ (por ejemplo, si $D$ contiene la oración " $F a$ ", entonces la oración " $F a$ v $F b$ " vale en $D) .4$

La definición de Carnap se inspira en la idea leibniziana de que una verdad necesaria es válida en "todos los mundos posibles"; y como en su sistema semántico $S_{1}$ los mundos posibles se hallan representados por las descripciones de estado, la definición de verdad lógica es fácil de adivinar:

Definición 2-2. Una oración $A$ es L-verdadera (en $S_{1}$ ) si y sólo si $A$ vale en todas las descripciones de estado.

Obsérvse que, a diferencia de la caracterización de "necesario" en términos de "mundos posibles", la definición 2-2 no incluye ningún concepto modal, cuya presencia podría hacer cuestionable su valor elucidatorio ("posible" no posee, en efecto, una claridad mayor que "lógicamente verdadero" o "necesario"). Aunque las decripciones de estado mencionadas en 2-2 corresponden o pretenden corresponder a la idea intuitiva de 'mundo posible', ni 2-2 ni la

2 El agregado entre corchetes señala un contraste obvio con el lenguaje natural, donde tales reglas _ en la medida en que existen - deben abstraerse de la conducta verbal de los hablantes.

3 Que Carnap denomine "convención" a su criterio de adecuación 2.1 no es un mero detalle, sino el signo seguro de su vocación convencionalista, vacilante a veces pero nunca abandonada. Otro aspecto del convencionalismo de Carnap se examina en la sección final de este trabajo.

4 Si bien el punto es trivial, recuérdese que $D$ es verdadera si y sólo si lo son todos sus miembros. 
definición misma de "descripción de estado" emplean la noción de posibilidad (o cualquier otra noción modal). ${ }^{5}$

He aquí, de acuerdo con Carnap, una reconstrucción exacta de lo que Leibniz entendía por "verdad necesaria" y Kant por "verdad analítica". Las vaguedades de Leibniz y Kant habrian sido reemplazadas, al fin, por la claridad de la semántica moderna, con su poderoso simbolismo, sus exquisitas letras góticas, sus constanes, firmemente adheridas a los objetos del universo, ${ }^{\circ}$ y sus versátiles variables. Pero ¿se ajusta la definición 2-2 a los requerimientos del criterio de adecuación?

Carnap no lo prueba de modo general, pero formula algunas consideraciones que "muestran - según dice textualmente- que el concepto de verdad-L aś definido satisface la condición $2-1 "(M \& N$, p. 11):

Si $A$ vale en toda descripción de estado, entonces las reglas semánticas de rango bastan para establecerlo (por ejemplo, las reglas semánticas nos dicen que " $P a$ " vale en ciertas descripciones de estado, que " $\sim P a$ " vale en todas las restantes, y que por lo tanto " $P a \mathrm{v} \sim P a$ " vale en toda descripción de estado). De allí que las reglas semánticas establezcan también la verdad de $A$, porque si $A$ vale en toda descripción de estado, entonces vale también en la descripción de estado verdadera. $(M \& N$, p. 11)

Sin embargo, todo lo que ha mostrado Carnap es que las reglas semánticas permiten establecer la verdad de la fórmula " $\mathrm{Pa} \sim \sim \mathrm{Pa}$ ", lo cual no es nada extraordinario; peró no mostró que si una fórmula arbitraria $A$ vale en cualquier descripción de estado (o sea: si es L-verdadera) entonces las reglas semánticas bastan para establecerlo. Si entendemos el verbo "establecer" en el sentido de "determinación efectiva" para todo caso particular, este hecho sería sorprendente, en vista de la indecidibilidad de la lógica de predicados.

Pero tal interpretación no es inevitable: podría decirse que lo que Carnap debe probar es que las reglas semánticas, consideradas como definiciones, implican lógicamente todas las oraciones verdaderas de la forma " $A$ es

5 Esto no impide, sin embargo, que la definición de descripción de estado se construya aplicando criterios lógicos y modales; ninguna descripción de estado puede contener, por ejemplo, una oración atómica y su negación, pues tal conjunción es lógicamente imposible. La aplicación de tales criterios ha dado origen al cargo de circularidad; pero es bueno advertir que tal circularidad - aun en el caso de que exista- no es atribuible a la definición misma.

6 Esta frase pretende ser una alusión irónica a cierta forma de fetichismo semántico, según el cual las reglas de designación juegan el mismo papel que Dios en la historia blblica. Un claro ejemplo es la fantástica afirmación de que "en un sistema semántico la relación de designación conecta a los signos con realidades objetivas" (H. Bohnert, "Carnap on definition and analicity", en P. A. Schilpp (ed.), The Philosophy of Rudolf Carnap, p. 413). Si se tiene en cuenta que una regla de designación de un sistema semántico en el sentido usual puede tener la forma " ' $\mathrm{X}$ ' designa el número 5 " (o " ' $\mathrm{X}$ ' designa al demonio que tentó a Lutero"), se comprenderá el poder demiúrgico que le confiere Bohnert. 
L-verdadera", aunque la prueba no sea efectiva. Ello requeriria: a) formalizar el metalenguaje $M$; $b$ ) definir 'consecuencia lógica' en $M$. En otros términos, se debe probar que son verdaderos en el metalenguaje todos los condicionales de la forma:

$A$ es L-verdadera en $S_{1} \supset\left(R \mid-\right.$ " $A$ es L-verdadera en $S_{1}$ "), donde " $R$ " representa el conjunto de las reglas semánticas y "|-" la relación de deducibilidad.

Volviendo ahora a nuestra observación anterior, no está de más insistir en que el texto de Carnap y los rasgos peculiares de su semántica sugieren con fuerza una interpretación menos amplia del verbo "establecer". Pues Carnap identifica el significado de una oración (su intensión) con su "rango lógico', o sea la clase de descripciones de estado en las que la oración vale ( $M$ \& $N, \S 40$ ); por otra parte, "comprender" una oración es "captar" su intensión: "El concepto de intensión se refiere al significado [meaning] en sentido estricto: aquello que captamos [which is grasped] cuando comprendemos [when we understand] una expresión" ( $M \& N$, p. 125). Además, "lo que un designador [p. ej., una oración (T. M. S.)] transmite del hablante al oyente es la intensión; es esta última la que el oyente comprende" ( $M$ \& $N$, p. 157). En consecuencia, si comprender una oración es captar su intensión, y ésta es una clase de descripciones de estado, entonces un hablante idóneo de $S_{1}$ (o sea un individuo exótico que adquirió un dominio completo de sus reglas semánticas) debe saber cuál es esa clase. Las verdades lógicas no pueden ser una excepción.

Finalmente, debido a que Carnap adopta la equivalencia lógica como criterio de identidad de intensiones, quien comprende dos oraciones matemáticas Lrequivalentes capta en ambos casos la misma intensión; de donde surge, o parece surgir, que debe conocer tal equivalencia sin la mediación de laboriosas demostraciones. Esto implica que o bien los teoremas de equivalencia son superfluos, o bien los matemáticos no comprenden las oraciones cuya equivalancia establecen. ${ }^{7}$

\section{Mundos posibles: perplejidades ontológicas}

Con el objeto de señalar más claramente algunos problemas relacionados con la elucidación carnapiana de 'mundo posible', construiremos la maquette

7 La vaguedad no aclarada de los términos "captar" y "comprender" podría servir de apoyo a una maniobra defensiva; frente a esta posibilidad, me acojo a los beneficios de un argumentum ad verecundiam: "Al basar el concepto de intensión en el de verdad-L surge una discordancia con la idea ... de que la intension 'se refiere al significado en sentido estricto: aquello que captamos cuando comprendemos una expresión'. Bien podria ocurrir, por ejemplo, que comprendiéramos el enunciado de un teorema aritmético -que según Carnap seria L-verdadero - sin saber cómo establecer su verdad o incluso sin saber que es verdadero" (Paul Bernays, reseña de Meaning and Necessity, en The Joumal of Symbolic Logic, vol. 14, no 4 (1949), pp. 287-41). 
ontológica correspondiente a un lenguaje más manejable que $S_{1}$, lenguaje al que podemos llamar " $L$ ". Supongamos que $L$ posee sólo dos constantes individuales, " $a$ " y " $b$ ", y dos predicados primitivos, " $F$ " y " $G$ ", de modo que se pueden formar a lo sumo cuatro oraciones atómicas: " $F a$ ", " $G a$ ", " $F b$ " y " $G b$ ". Hay, pues, 16 descripciones de estado diferentes, que representan todos los estados posibles del universo de individuos con respecto a las propiedades y relaciones expresadas por los predicados de L. Al desplegar estos mundos en una tabla análoga a las tablas veritativas, pondremos cada oración atómica en lugar de la "V" de "Verdadero" y sus negaciones en lugar de la "F" de "Falso"; para mantener la similitud con las tablas usuales, las columnas estarán encabezadas por las letras " $p$ ", " $q$ ", " $r$ " y " $s$ ". La tabla que obtenemos es entonces la siguiente:

$\left.\begin{array}{rrrrc} & p & q & r & s \\ \hline 1 & F a & F b & G a & G b \\ 2 & F a & F b & G a & \sim G b \\ 3 & F a & F b & \sim G a & G b \\ 4 & F a & F b & \sim G a & \sim G b \\ 5 & F a & \sim F b & G a & G b \\ 6 & F a & \sim F b & G a & \sim G b \\ 7 & F a & \sim F b & \sim G a & G b \\ 8 & F a & \sim F b & \sim G a & \sim G b \\ 9 & \sim F a & F b & G a & G b \\ 10 & \sim F a & F b & G a & \sim G b \\ 11 & \sim F a & F b & \sim G a & G b \\ 12 & \sim F a & F b & \sim G a & \sim G b \\ 13 & \sim F a & \sim F b & G a & G b \\ 14 & \sim F a & \sim F b & G a & \sim G b \\ 15 & \sim F a & \sim F b & \sim G a & G b \\ 16 & \sim F a & \sim F b & \sim G a & \sim G b\end{array}\right\}$

Esta tabla muestra algunos detalles interesantes: 
i) La fila 1 parece trivial, pero encierra cierta dosis de misterio. Si pensamos que cada descripción de estado describe un mundo posible, entonces advertimos que en este mundo todos los individuos tienen las mismas propiedades y por lo tanto son indiscernibles, pese a lo cual siguen siendo numéricamente diferentes. En efecto, $a$ es $F$, y también lo es $b ; a$ es $G$, y también lo es $b$; y no hay ninguna propiedad de $b$ que no sea propiedad de $a$. En rigor, esto es incompatible con la definición leibniziana de identidad, que contiene como parte el principio de identidad de los indiscernibles:

$$
\text { (F) }(F x \equiv F y) \supset(x=y) \text {. }
$$

Sin embargo, parece claro que Carnap no rechaza este principio; en la página ${ }_{14}$ de $M \& N$ leemos que si se agrega a $S_{1}$ una variable predicativa, entonces la identidad es definible a la manera de Leibniz-Russell. ${ }^{8}$

ii) El individuo $a$ tiene en el mundo posible 6 todas las propiedades de $b$ en 11, y $b$ posee en 11 todas las propiedades de $a$ en 6 . Pero ni $a$ ni $b$ pierden su identidad pese a que han intercambiado todas sus propiedades. Estas son los fugaces trajes de las sustancias, que al cambiar de mundo se quitan a veces la corbata y la camisa, y otras se cambian la ropa entera. Estamos, al parecer, frente al sustrato incognoscible de Locke, que subyace a todas las propiedades.

iii) Además, los mundos 6 y 11 son lógicamente distintos pero empíricamente indistinguibles. Desde el punto de vista empírico describen exactamente el mismo mundo. Esto es lo que ha llamado Neil Wilson "la paradoja de las descripciones de estado" y que podríamos llamar "la paradoja de Carnap" o "el colmo del empirismo". Según observa Wilson, esta situación es el resultado de una concepción simplista acerca de las relaciones del lenguaje con el mundo: es la idea de que los predicados se "agarran" a las propiedades, y los nombres propios a los individuos simples o trozos de sustratos. Wilson propone un análisis realista de los nombres propios en virtud del cual las

8 Podría objetarse que en la tabla no están representadas todas las propiedades, lo cual deja abierta la posibilidad de que en el mundo 1 exista al menos una propiedad $H$ que $a$ y $b$ no poseen en común; de acuerdo con esta suposición, la fila i sólo sería la descripción parcial de un mundo posible. Pero, en primer lugar, las descripciones de estado de Carnap no pretenden ser descripciones parciales sino descripciones completas relativas a un lenguaje dado: representan la noción de 'mundo posible respecto de un lenguaje $L$ '. En segundo lugar, el problema planteado no depende en absoluto de la capacidad expresiva del lenguaje, sino de las reglas según las cuales se construyen las descripciones de estado, reglas que son formalmente idénticas a las que orientan la construcción de tablas veritativas. Asi como en toda tabla veritativa exite una fila que sólo contiene la letra " $\mathrm{V}$ ", independientemente del número de oraciones atómicas consideradas (que podráa ser infinito), las correspondientes tablas de mundos posibles deben contener una descripción de estado en la que figuren todas las oraciones atómicas y ninguna de sus negaciones, aunque haya una infinitud de predicados primitivos (en cuyo caso no será representable in toto mediante un gráfico visible, pero Dios la contemplará sin esfuerzo). Cabe recordar que el número de constantes individuales de $S_{1}$ es infinito. 
descripciones 6 y 11 resultan ser lógicamente equivalentes y la paradoja se desvanece: describen, en verdad, el mismo mundo.` Claro que ello requiere un análisis cuidadoso de la función designativa; hace falta algo más que las definiciones por enumeración que encontramos en la semántica formal al estilo de Carnap.

iv) Finalmente, consideremos la descripción de estado número 16. Presenta un mundo desolador de meras sustancias carentes de propiedades. Desde el punto de vista formal podría replicarse que " $\sim F^{\prime \prime}$ y " $\sim G$ " son predicados legítimos y por lo tanto también expresan una propiedad. Si decimos que César no era ruso ni chino, sin duda decimos algo sobre César; ocurre que en realidad era romano. Pero si afirmamos que no era alto, ni bajo, ni de estatura mediana, ni de ninguna altura, podemos preguntarnos si la palabra "César" se refiere a alguien que sea algo más que una mera sustancia. Dicho en forma general: si dada una lista completa de los predicados primitivos de un lenguaje sucede que ninguno de ellos es aplicable a César, ¿qué sentido tiene pregonar que César posee -relativamente a ese lenguaje- sólo pro piedades negativas, y en qué se diferencia esto de decir que no tiene propiedad alguna? 10

Si convenimos pues, en que 6 pretende describir un mundo de sustancias puras, podría sugerirse que tal mundo es lógicamente imposible. La respuesta final depende, naturalmente, de lo que entendamos por "posibilidad lógica": ni el mismo Carnap -como veremos pronto- aceptaría reducirla a las combinaciones gramaticalmente posibles de constantes y signos de negación en las filas de una matriz.

\section{Predicados no independientes; división del campo de lo analitico}

Pero aun dejando de lado lo anterior, ces verdad que cada descripción de estado representa un mundo posible relativo a nuestro mini-lenguaje? Para construir la tabla hemos seguido un criterio puramente combinatorio: las 16 filas constituyen todas las descripciones de estado que se pueden obtener combinando las cuatro oraciones atómicas y sus negaciones, independientemente del contenido de los predicados y con la única restricción de que ninguna fila púede contener dos oraciones de la forma $A$ y $\sim A$. En cuanto a los predicados " $F$ " y " $G$ ", ni siquiera nos tomamos la molestia de darles una interpretación. $Y$ esto nos pareció muy natural, pues siempre se ha dicho que la verdad lógica es puramente "formal" (sólo depende del significado $\rightarrow$ de las reglas de uso- de los términos lógicos, considerados también "formales") y que lo mismo es válido para las nociones correspondientes de

- Neil L. Wilson, "Substances without Substrata", The Review of Metaphysics, 12 (1959); incluido en M. Bunge, ed., Antologia semántica, Bs. As, (1960).

10 La nota 8 es pertinente aquí. 
posibilidad e imposibilidad. Desde este punto de vista no hay nada objetable en la tabla de mundos posibles, pues ninguna fila contiene una inconsistencia "formal", o sea, dos oraciones relacionadas como $A$ y $\sim A$.

Supongamos ahora que los predicados " $F$ " y " $G$ " reciben la siguiente interpretación: $\boldsymbol{F}=$ soltero y $\mathbf{G}=$ casado. La primera fila de la tabla representa entonces un mundo 'posible' en que el individuo $a$ (y también $b$ ) es casado y no casado), ya que "soltero" es sinónimo de "no casado". Esta es la objeción que Quine presentó en "Two Dogmas of Empiricism": "El criterio de analiticidad en términos de descripciones de estado sólo sirve para lenguajes desprovistos de pares de sinónimos extralógicos" (From a Logical Point of $V i e w$, p. 29). En este artículo Quine había dividido los enunciados analíticos en dos clases, ejemplificadas respectivamente por

1) Ningún hombre no casado es casado

y

\section{2) Ningún soltero es casado.}

De acuerdo con su propuesta terminológica, los enunciados analíticos de la primera clase son "lógicamente vendaderos"; los de la segunda son "analfticos" (simpliciter) pero no expresan verdades lógicas. La verdad de 1) depende sólo del significado de los términos lógicos, y por lo tanto sobrevive a cualquier variación de sus componentes descriptivos; 2), en cambio, se distinguen por el hecho de que su verdad depende no sólo del significado de sus signos lógicos, sino también del de sus constantes descriptivas; ${ }^{11}$ posee, además, la peculiaridad de que puede convertirse en un enunciado lógicamente verdadero mediante el intercambio de sinónimos. Así, 2) se convierte en 1) si remplazamos "soltero" por "no casado". Un enunciado analítico es, pues, de acuerdo con la sugerencia de Quine, o una verdad lógica (en el sentido usual), o un enunciado transformable en una verdad lógica mediante sustituciones sinonímicas. El resultado neto es que "el criterio en términos de descripciones de estado es a lo sumo una reconstrúcción de la verdad lógica, pero no de la analiticidad [en general]" (loc. cit., pp. 23-24).12

11 Obsérvese que, contrariamente a la forma lógica de 1), la de 2) no es válida.

12 En contraste con la noción de verdad lógica, a la que no juzga problemática, Quine piensa que no hay - ni puede haber - una caracterización adecuada de analiticidad en general. La caracterización anterior,' presentada por Quine con fines polémicos, se basa en la relación de sinonimia, que Quine considera ininteligible.

Esta brecha tajante sugiere una digresión. La definición de verdad lógica depende de una condición esencial: "Si suponemos una lista previa de las partículas lógicas -escribe Quine- [...] entonces [...] una verdad lógica es un enunciado que es verdadero y sigue siéndolo bajo todas las interpretaciones de sus componentes no lógicos" (loc. cit., pp. 22-23). La definición es conocida y previsible. Pero nadie ha dado nunca una caracterización satisfactoria de "partícula lógica" aplicable a un lenguaje especifico, y, menos aún, a cualquier lenguaje. La claridad del concepto de verdad lógica se halla limitada, pues, por la oscuridad del concepto de 'signo lógico' en que se apoya; podemos ofrecer una lista -inevitable- 
En rigor, la restricción que tendríamos que imponer a un lenguaje para salvar la definición de Carnap es mucho más fuerte de lo que dice Quine: al dividir los enunciados analíticos en dos clases Quine se olvidó de enunciados como

3) Si $x$ es rojo, entonces $x$ es coloreado,

que no puede convertirse en una verdad lógica mediante ninguna artimaña con sinónimos. De modo que enfrentariamos el mismo problema si interpretáramos " $F$ " como "rojo" y " $G$ " como "coloreado". Pero la dificultad es de más largo alcance: pruébese interpretar " $F$ " como "amarillo" y " $G$ " como "azul", o " $F$ " como el predicado relacional "más alto que"; en este último caso habria una descripción de estado que contendría tanto " $F(a, b)$ " como " $F(b, a)$ ", pese a que la relación es asimétrica. Y si agregamos a nuestro minilenguaje una constante individual " $c$ ", entonces podriamos construir un mundo 'posible' en que se dieran $F(a, b), F(b, c)$ y $\sim F(a, c)$, a pesar de que con la interpretación asignada $F$ es transitiva: nada hay en la definición de descripción de estado que impida estos escándalos.

\section{Postulado de significación: verdades lógicas directas}

Al examinar esta situación en su artículo "Meaning Postulates" (que figura como apéndice en la 2da. ed. de $M \& N$ ) Carnap habla de las "relaciones lógicas entre los significados atribuidos a los predicados primitivos" (p. 224; la bastardilla es mía) y admite que en virtud de ellas ciertas descripciones de estado "no representan estados posibles" (p. 227). Merece observarse que esto constituye un abandono de la idea de que las relaciones 'lógicas' son meramente formales, al menos en el sentido de que dependen exclusivamente del vocabulario lógico; la 'materia' de los enunciados, o sea el significado de las constantes descriptivas, determina también la existencia de tales relaciones y es relevante para la noción de posibilidad lógica. ${ }^{13}$ Carnap coincide

mente arbitraria - de signos lógicos, pero no una definición general. Gracias a esta dificultad, la amplitud de la brecha abierta por Quine parece reducirse. Pues podríamos proclamar tạmbién, a la Quine, que "si suponemos una lista previa de sinónimos extralógicos, entonces una verdad analítica es un enunciado que...", etcétera.

Quine impugna igualmente la relativización carnapiana de la noción de analiticidad a un lenguaje artificial especifico $L_{0}$ : "Al decir qué enunciados son analíticos en $L_{0}$ explicamos 'analítico-en- $L_{0}$ ' pero no 'analítico' [en general]" ("Dogmas", p. 33). Sin embargo, la misma objeción es claramente aplicable a la definición quineana de verdad lógica, ya que la lista de signos lógicos es siempre relativa a un lenguaje particular. Para un rechazo explícito de la simetria de ambas situaciones véase W. V. O. Quine, "Carnap on Logical Truth", \& IX.

is Este uso del adjetivo "Iógico" es introducido en M\&N de manera algo subrepticia a través de la convención 2-1, que hace depender la verdad lógica de las reglas semánticas, las que incluyen las reglas de designación; 2-1, combinada con la observación inicial de que usará las expresiones "Humano" y "Animal Racional" como sinónimas (p. 4), le permite inferir a Carnap en la p. 15 que la oración " $X$ es humano $\supset X$ es un animal racio- 
ahora con Quine en la conveniencia de dividir los enunciados analíticos (es decir, los "verdaderos en virtud del significado solamente") en las dos clases ejemplificadas por 1) y 2).

Para que la noción de analiticidad se aplique también a los ejemplos heterodoxos, y, en general, a los casos en que hay "relaciones lógicas entre los predicados primitivos del sistema" (p. 224), se deben introducir en él postulados especiales que formulen explícitamente tales conexiones. Como veremos en detalle, este es un modo de anular al enemigo invitándolo a dormir en casa e imponer sus normas de convivencia. Un ejemplo de postulado de significación es el siguiente:

$$
\left(P_{1}\right)(x)(S x \supset \sim C x)
$$

donde " $S$ " significa soltero y " $C$ ", casado. Carnap aclara que "este uso de 'postulado' no es el mismo ... según el cual 'postulado' es sinónimo de 'axioma'" (p. 224), sin duda con la intención de dejar bien en claro que un postulado de significación carece de contenido fáctico, al igual que una tautología. La advertencia es igualmente válida para posibles postulados como:

$$
\begin{aligned}
& \left(P_{2}\right)(x)(x \text { es rojo } \supset x \text { no es azul) } \\
& \left(P_{8}\right)(x)(x \text { es rojo } \supset x \text { es coloreado) } \\
& \left(P_{4}\right)(x)(x \text { es un cuervo } \supset x \text { es negro), }
\end{aligned}
$$

que indicarían simplemente la decisión de usar los predicados del sistema de modo tal que ciertas situaciones queden excluidas; y no hay en el concepto de postulado de significación restricción alguna que impida introducir bajo este nombre una estipulación según la cual dos predicados arbitrarios guardan entre sí determinadas relaciones de consecuencia lógica $o$ incompatibilidad.

La definición de analiticidad que resulta de introducir postulados de significación puede formularse de varios modos, pero la idea esencial es simple: un enunciado es analítico si y sólo si vale en todas las descripciones de estado en que valen los postulados de significación. Podemos llamar a estas últimas "descripciones admisibles", y decir, más brevemente, que una oración es analítica si y sólo si vale en todas las descripcionès admisibles. Este procedimiento es presentado de manera algo más tajante diciendo que "limita las descripciones de estado a las que representan casos posibles mediante postulados de significación o reglas (sic) equivalentes" (p. 223). Es claro que de este modo se preserva también la validez de las leyes lógicas "en sentido

nal" es lógicamente verdadera, pese a que su forma lógica es, en sentido estricto, la de un enunciado contingente, y por lo tanto no vale en todas las descripciones de estado, hecho que Carnap no advirtió cuando escribla Meaning and Necessity. 
restringido" [in the narrow sense] como dice Carnap (p. 223), pues si $A$ vale en todas las clases de oraciones que considerábamos antes descripciones de estado, entonces debe valer en las descripciones admisibles, que son ahora, para el lenguaje enriquecido con los postulados, las descripciones a secas.

La referencia a la verdad lógica en sentido restringido implica que los enunciados analíticos, y, en particular, los postulados de significación mismos, son también verdades lógicas, aunque en sentido amplio. Por eso dice Carnap (p. 227) que la desventaja de este procedimiento consiste en requerir la introducción de un nuevo concepto semántico: el de "L-verdadero de manera directa, es decir, postulado de significación". Como observa R. M. Martin (en Paul A. Schilpp, The Philosophy of Rudolf Carnap, p. 382) la noción de verdad-L no recibe ahora un análisis $a b$ initio, sino que se reduce al de 'verdad lógica directa', que es un concepto semántico primitivo.14

\section{Esopo y la semántica: el misterio de las propiedades simples}

Cabe señalar que la mayor parte de los predicados que obligan a efectuar estas reformas (p. ej., "Rojo" y "Coloreado") parecen expresar propiedades simples, de modo que no queda esperanza de que las relaciones entre ellos que Carnap denomina "Iógicas" puedan reducirse por análisis a otras de carácter más ortodoxo, en las que ningún término descriptivo figure esencialmente.

Introduciré ahora una breve fábula, cuya moraleja dejo a cargo del lector. Hace ya mucho tiempo hubo alguien que enfrentó un problema similar al de Carnap, y a quien la relación de incompatibilidad entre las propiedades simples produjo un estado de insoluble perplejidad. "El hombre no sabe todavía -escribió Leibniz - cuál es la razón de la incompatibilidad de cosas diferentes, o cómo ocurre que diferentes esencias puedan oponerse entre sí, siendo que todos los términos puramente positivos parecen compatibles" (Gerhardt, t. VII, p. 195). "Rojo" y "Azul" son un ejemplo de tal incompatibilidad, y sin embargo ninguno de estos términos es la negación formal del otro, como requeriría el logicismo de Leibniz. Varios siglos después otro hombre, Wittgenstein, tomó nota del asunto y decretó simplemente que " $x$ es rojo" $y$ " $x$ es azul" son lógicamente contradictorios; ${ }^{15}$ esto puede sorprender

14 Para apreciar en toda su magnitud la transformación sufrida por los adjetivos "16gico" y "analítico" basta notar que desde el punto de vista de Husserl $\left(P_{2}\right)$ y $\left(P_{3}\right)$ expresarían proposiciones sintéticas a priori, cuya validez reposa en la peculiaridad de las 'esencias materiales' Rojo, Azul y Coloreado; son leyes sintéticas que valen con 'universalidad esencial' en una 'ontologia regional' determinada. En cambio la validez de las proposiciones analiticas sólo depende de las conexiones necesarias entre esencias meramente formales (Objeto, Propiedad, Relación, Todo; Parte, etc.). Cfr. Investigaciones lógicas, t. III, Investigación III, 11 y 12.

1s "Así como sólo existe la necesidad lógica, sólo hay, también, imposibilidad lógica. Por ejemplo, que dos colores estén en el mismo lugar en el campo vísual es imposible, es 
pero en realidad no hay de qué preocuparse, pues como ha observado el mismo Wittgenstein, al fin y al cabo "los objetos carecen de color" (Tractatus, 2.0232). Detrás de Wittgenstein llegó Rudolf Carnap, quien al advertir que la cuestión era molesta estipuló que todo se arregla con estipular que " $x$ es rojo y $x$ es azul" es una falsedad lógica directa. Leibniz era, pues, un hombre que dudaba; Wittgenstein, por el contrario, no encontró motivo alguno para la duda, y Rudolf Carnap, que ni dudaba ni estaba convencido, pensó que lo mejor era que cada uno pensara lo que quisiera con tal de que lo consignara de modo categórico en un sistema semántico $S_{i}$.

Esta úlima postura es una versión caricaturesca del convencionalismo, y debo reconocer que no es del todo justa con respecto a Carnap; cuál es, medida en miligramos, la proporción exacta de ese ingrediente en la semántica de Carnap es algo que veremos luego con mayor detalle. Pueden encontrarse en Carnap claras manifestaciones anticonvencionalistas, y aun esencialistas, particularmente en su polémica con Quine; pero el convencionalismo constituye sin embargo uno de los polos entre los que oscila el pensamiento carnapiano.

\section{Analiticidad y reglas de designación}

En "Meaning Postulates" Camap sostiene que la analiticidad puede definirse antes de dar una interpretación de las constantes descriptivas. En efecto, después de introducir el postulado $\left(P_{1}\right)$ dice textualmente:

Aun ahora no damos reglas de designación para " $S$ " y " $C$ ". No son necesarias en la elucidación de la analiticidad, sino solo en la elucidación de la verdad fáctica. Pero el postulado $\left(P_{1}\right)$ dice acerca de los significados de " $S$ " y " $C$ " todo lo que se requiere para la analiticidad, a saber: que las dos propiedades son incompatibles (loc. cit., p. 224).

decir, lógicamente imposible, puesto que se halla excluido por la estructura lógica del color" (Tractatus, 6.375-6.3751). En el mismo versiculo Wittgenstein llama al enunciado sobre la presencia de dos colores en el mismo campo visual una "contradicción". El lector advertirá que la afirmación de Wittgenstein se apoya en un uso malabar de las palabras "contradicción" y "lógica"; se atribuye la imposibilidad a "la estructura lógica del color" (?), aunque tal imposibilidad no sea reducible a una contradicción formal (del tipo simbolizado por " $p \cdot \sim p$ "). En una época posterior el autor del Tractatzus adoptó una posición más cautelosa: "Las proposiciones atómicas, aunque no pueden contradecirse, pueden excluirse entre si" ("Some Remarks on Logical Form", The Aristotelian Society, Supplementary volume ix, 1929, pp. 162-171, esp. p. 168; la bastardilla es m(a). La cita es de William Kneale, quien comenta: "Esta admisión es fatal a la doctrina de su obra anterior, pero Wittgenstein se hallaba aún tan influido por su propio uso laxo [de los términos] que trató de presentar este cambio como una reforma de la lógica y no como un abandono de la tesis de que no hay imposibilidad fuera de la lógica" (William and Martha Kneale, The Development of Logic, Oxford Clarendon Press, p. 634; hay trad. cast. de Javier Muguerza: Tecnos, Madrid). Sobre las mencionadas acrobacias de Wittgenstein véase también A. Pap, Semantics and Neccesary Truth, Yale University Press, 1958, cap. 5, p. 102 (hay trad. cast. de Nicolás Molina Flores: Semántica y verdad necesaria, Fondo de Cultura Económica, México). 
Según esto sabríamos que $\left(P_{1}\right)$ es una verdad lógica directa aunque " $S$ " y " $C$ " no tengan significado alguno; la asignación de significados sería después un asunto de mera decisión. Hay sin embargo motivos para la duda: yo puedo saber, por ejemplo, que " $S$ " y " $C$ " se usan como sinónimos y por lo tanto que " $(x)(S x \equiv \sim C x)$ " es analítico aunque ignore los significados de " $S$ " y " $C$ "; pero " $(x)(S x \equiv \sim C x)$ " no es una verdad analítica si los predicados no designan nada. No siendo verdadero (ni falso), tampoco es analiticamente verdadero. En el caso particular de un sistema semántico, mientras no se hayan formulado las reglas de designación el postulado $\left(P_{1}\right)$ es sólo una promesa de analiticidad.

\section{Analiticidad y contenido fáctico}

Examinemos ahora con más cuidado la manera en que Carnap formula el contraste entre oraciones analiticas y sintéticas: la verdad de las primeras "se basa en el significado"; la de las segundas, en cambio, "depende de los hechos del mundo" ("Meaning Postulates", p. 222). En el primer caso "es suficiente comprender el enunciado para establecer su verdad; no se requiere el conocimiento de hechos extralingüísticos" (loc. cit.)

Carnap dice aquf dos cosas:

a) que la verdad de un enunciado analítico "se basa" en el significado;

b) que basta comprender su significado para establecer su verdad. ${ }^{16}$

El texto permite suponer que $a$ ) y b) son de algún modo propiedades equivalentes de los enunciados analfiticos. Como Carnap sostiene - fiel a la tesis central del empirismo lógico - que todo lo necesario es analítico, esta caracterización es un paso importante en la explicación de la necesidad, que debe hacer imposible la existencia de juicios sintéticos a priori. ${ }^{17}$ Suele admitirse que la necesidad analítica no posee el carácter problemático de la que se presenta como sintética; Kant no se preguntó: "¿Cómo son posibles los juicios analf́ticos?”, sino: “¿Cómo son posibles los juicios sintéticos a priori?". La clave se halla en que, de acuerdo con Kant, los juicios analíticos "no extienden nuestro conocimiento". En consecuencia, a) y b) deben implicar:

16 Recuérdese que en la sección 2 pusimos en duda la validez general de (b), dada la extensión conferida por Carnap al término "analltico". Es interesante mostrar cierto paralelismo con una objeción de Frege a Kant, vinculada con la ausencia de una intuición a priori en la que pudiera apoyarse la suma de números grandes (Cfr. G. Frege, The Foundations of Arithmetic, Philosophical Library, N. Y., 1950, p. 6e).

17 "Según lo señaló una vez Moritz Schlick, el empirismo puede ser definido como el punto de vista de acuerdo con el cual lo sintético a priori no existe. Si es posible reducir todo el empirismo a una fórmula, esta es la única manera de hacerlo" (R. Carnap, Fundamentación lógica de la física, ed. Sudamericana, Bs. As., 1969, p. 241). 
c) Un enunciado analítico carece de contenido fáctico (no transmite información sobre el mundo).

Esto es, en efecto, lo que piensa Carnap. La amalgama de $(a),(b)$ y $(c)$ se hace claramente visible en otro texto:

[la oración 'Ningún soltero es casado'] [...] es verdadera no a causa de la naturaleza del mundo sino de los significados que nuestro lenguaje asigna a las palabras descriptivas [...] La verdad o falsedad de una oración sintética, en cambio, no está determinada por los significados de los términos sino por la información fáctica acerca del mundo. Por ejemplo, "Los objetos caen a tierra con una aceleración de $980 \mathrm{~m} / \mathrm{s}$ "; no es posible determinar si este enunciado es verdadero o falso simplemente mediante el examen del significado. Hay que realizar una prueba empírica. Tal enunciado tiene contenido fáctico. (Fundamentación de la fisica, pp. 345-346).

¿Por qué el hecho de que para establecer la verdad de una oración analltica sea suficiente conocer su significado muestra su carencia de contenido fáctico? En otros términos: (por qué $b$ ) implica $c$ )? Prima facie, b) es perfectamente compatible con la negación de $c$ ). Si no se aportan otros argumentos, el paso de b) a c) es una mera petición de principio; presupone una conexión no probada entre el modo de conocer la verdad de las oraciones y sus propiedades puramente semánticas, que podrían ser independientes de las características del proceso cognoscitivo.

Pero encontramos en Carnap otro modo de defender $c$ ), que vale la pena examinar. Su ejemplo es un posible postulado de significación que establece la asimetría de la relación ser más caliente que: " $(x)(y)$ (Si $x$ es más caliente que $y$, entonces $y$ no es más caliente que $x)^{\prime \prime}$ :

Si alguien nos dice que ha descubierto dos objetos $A$ y $B$ tales que $A$ es más caliente que $B$ y $B$ más caliente que $A$, no responderíamos: 'QQué sorprendentel 'Qué maravilloso descubrimiento!' Más bien responderíamos: 'Usted y yo debemos interpretar de manera distinta la expresión "más caliente". Para mí significa una relación asimétrica. Por ló tanto, la situación que usted halló no puede describirse como usted lo ha hecho'. El postulado [de significación] se refiere exclusivamente al significado de la palabra tal como se lo usa en nuestro lenguaje. No dice nada sobre la naturaleza del mundo (loc. cit., p. 350).

Este pasaje exhibe un sorprendente non sequitur. Como ha observado agudamente Quine, cuando alguien sostiene algo imposible, o niega una verdad obvia, es razonable pensar que está empleando las palabras con un significado insólito: "No puede haber prueba más fuerte de un cambio de significado que el repudio de lo obvio" ("Carnap on logical truth", en Schilpp, 
p. $3^{89}$ ); pero esto no implica que la oración negada carezca de contenido fáctico. ${ }^{18}$ Interesa señalar que la observación es también aplicable a oraciones que no nos intimidan con el augusto resplandor de la verdad a priori; si en medio de una lluvia torrencial un hombre calado hasta los huesos se empeña en negar que está lloviendo, sería natural que le dijéramos: "Oiga, ¿qué entiende usted por 'llover' ?".

\section{El traspié convencionalista}

Llegamos por fin al problema del convencionalismo.

Supongamos - escribe Carnap- que el autor de un sistema desea que los predicados " $S$ " y " $M$ " designen las propiedades Soltero y Casado, respectivamente. ¿Cómo sabe que estas propiedades son incompatibles y que por eso debe introducir el postulado $\left(P_{1}\right)$ ? Esta no es una cuestión de conocimiento sino de decisión. Su conocimiento o creencia de que las palabras "soltero" y "casado" son entendidas usualmente de modo tal que ellas [las propiedades] son incompatibles, puede influir en su decisión si el autor tiene la intención de reflejar en su sistema algunas de las relaciones entre significados que se dan entre las palabras castellanas [inglesas]. En este caso particular, la influencia sería relativamente clara, pero en otros casos lo sería mucho menos. (Pp. 224-25; la bastardilla es mia).

Confieso que este párrafo tiene para mí cierta atmósfera surrealista. No entiendo cómo se puede decidir que las propiedades Soltero y Casado sean o no incompatibles. Podemos decidir qué propiedades designarán las constantes " $S$ " y " $G$ ", pero no qué relaciones existen entre las propiedades mismas. Después de todo, los postulados de significación se introdujeron debido a la existencia admitida de relaciones lógicas entre tales propiedades - que en Carnap ofician de significados-, y si es cuestión de decidir, también podriamos decidir que todos los predicados son independientes. El par "Soltero Casado" es además un caso paradigmático; si " $S$ " significa Soltero y " $C$ " significa Casado, ¿cómo puede Carnap —o Dios- decidir que un hombre casado puede ser soltero? Lo único que es posible decidir es usar las palabras con otro significado, como el mismo Carnap lo dice en su polémica con Quine.

Carnap no se equivoca, sin embargo, cuando se trata de predicados vagos, o sea de predicados respecto de los cuales no se sabe exactamente qué propiedad designan; por ejemplo, ¿'es posible que un cuervo sea violeta, o "cuervo" designa una propiedad compleja uno de cuyos componentes es la negrura? Sin duda hay aquí lugar para una decisión acerca del significado de "cuervo",

18 En "Logical necessity, physical necessity, ethics and quantifiers" (Inquiry, III, 1960, pp. 259-269), R. Montague sostiene que "el sentido amplio de necesidad lógica debe considerarse como una especie de necesidad fisica. Las 'leyes fisicas' involucradas son las que Carnap llama postulados de significación" (p. 265). 
pero una vez tomada la decisión acerca de la propiedad designada por este término, se acabó la posibilidad de decidir. ${ }^{18}$ Mi impresión es que en este artículo Carnap confundió ambos casos, lo que no hace cuando discute con Quine. Refiriéndose a la oración "Todos los perros negros son perros" dice textualmente:

Una vez que los significados de las palabras [...] están dados (lo que sí puede considerarse materia de convención) ya no es cuestión de convención o de elección arbitraria la de si debemos o no considerar tal oración como verdadera. La verdad de esta oración está determinada por las relaciones lógicas que valen entre los significados dados (en este caso sólo son relevantes los de "todos" y "son"). (Schilpp, p. 916)

Esta es una clara posición anticonvencionalista. Si tomamos en cuenta la noción de verdad lógica directa, que está determinada también —según Carnap- por las relaciones lógicas que valen entre los significados de las constantes descriptivas, entonces se concluye lo que sostuvimos antes: la relación lógica entre las propiedades Soltero y Casado no puede ser asunto de convención; una vez decidido que " $S$ " significa Soltero y " $C$ ", Casado, sólo nos queda reconocer -introduciendo un postulado de significación-que por desgracia las dos cosas son incompatibles. Carnap mismo dice en otra parte: "La equivalencia de propiedades no depende del lenguaje" ( $M \& N$, p. 24).

\section{Verdad lógica y cuantificación sustitucional}

El carácter sintáctico de la definición carnapiana de 'valer en una descripción de estado' determina de un modo especial la condición veritativa de las oraciones universales. De acuerdo con tal definición, una oración de la forma " $(x)(\ldots x \ldots)$ " vale en una descripción de estado si y sólo si valen también en ella todos los casos de sustitución de su alcance. Supongamos que el alcance tiene la forma simple " $F x$ ", donde " $F$ " es un predicado primitivo; entonces " $(x) F x$ " vale en una descripción de estado si y sólo si los infinitos enunciados atómicos de la forma " $F a_{1}$ ", " $F a_{2}$ ", . . , pertenecen a tal descripción de estado. Si ésta es la descripción de estado verdadera, resulta que " $(x) F x$ " es verdadera si y sólo si son verdaderos todos los casos de sustitución de su alcance. Pero, como se ha observado muchas veces, esto exige que haya tantas constantes individuales como objetos en el universo del discurso. En particular, el dominio de objetos de $S_{1}$ debe ser a lo sumo numerable. Sólo esta correspondencia entre objetos y constantes individuales permite lo que se denomina interpretación sustitucional de la cuantificación, en lugar de la

19 Hace poco vi en una revista la fotografía de un cuervo blanco. Era blanco sin duda alguna, ¿pero era realmente un cuervo? Los zoólogos han decidido que si, tomando como características definitorias de la especie propiedades que no incluyen el color. 
cuantificación objetal. Si no se cumple con este requisito, entonces la definición anterior podría impugnarse. Pues supongamos que el dominio de $\boldsymbol{x}$ son los números reales, que forman un conjunto no numerable. En tal caso " $(x) F x "$ podria valer en la descripción de estado verdadera y sin embargo ser falsa, si hubiera números reales que no poseen la propiedad $F$. En cambio, en la interpretación objetal de la cuantificación se dice, grosso modo, que " $(x) F x$ " es verdadera si y sólo si todo valor de $x$ es $F$, independientemente de que para cada valor de $x$ haya o no una constante individual que la represente en el lenguaje. El hecho de que los mundos posibles de Carnap se hallen constituidos por descripciones de estado impone, pues, una limitación muy severa al poder expresivo de sus sistemas semánticos; y ésta es la razón por la cual Carnap remplazó posteriormente la noción de descripción de estado por la de modelo, que es una entidad extralingüistica (Schilpp, p. 915). La relación de valer en una descripción de estado es remplazada ahora por la de ser verdadera en un modelo, lo que exige a su vez que las reglas veritativas adquieran una forma diferente: en lugar de: ' ' $F a$ ' es verdadera si y sólo si..." tendremos: “ ' $F a$ ' es verdadera en un modelo $W$ si y sólo si...". Finalmente, una oración es verdadera (simpliciter) si y sólo si es verdadera en el mundo real.*

Thomas M. Simpson

Consejo Nacional de Investigaciones Científicas y Técanicas de la República Argentina

- El presente trabajo fue presentado como ponencia al rer Coloquio Nacional de Filosofía realizado en Morelia, Mich., en agosto de 1975. 